\title{
Galactic cosmic rays and the knee - Results from the KASCADE experiment ${ }^{1}$
}

\author{
J.R. Hörandel*, W.D. Apel ${ }^{\dagger}$, F. Badea ${ }^{\dagger}$, K. Bekk ${ }^{\dagger}$, A. Bercuci**, \\ J. Blümer ${ }^{\dagger, *}$, H. Bozdog ${ }^{\dagger}$, I.M. Brancus ${ }^{* *}$, A. Chilingarian ${ }^{\ddagger}$, K. Daumiller ${ }^{\dagger}$, \\ P. Doll ${ }^{\dagger}$, R. Engel ${ }^{\dagger}$, J. Engler ${ }^{\dagger}$, H.J. Gils ${ }^{\dagger}$, R. Glasstetter, ${ }^{*}$, A. Haungs ${ }^{\dagger}$, \\ D. Heck ${ }^{\dagger}$, K.-H. Kampert ${ }^{*, \S}$, H.O. Klages ${ }^{\dagger}$, G. Maier ${ }^{\dagger}$, II , H.J. Mathes ${ }^{\dagger}$, \\ H.J. Mayer ${ }^{\dagger}$, J. Milke ${ }^{\dagger}$, M. Müller ${ }^{\dagger}$, R. Obenland ${ }^{\dagger}$, J. Oehlschläger ${ }^{\dagger}$, \\ S. Ostapchenko ${ }^{\dagger, \|}$, M. Petcu**, S. Plewnia ${ }^{\dagger}$, H. Rebel ${ }^{\dagger}$, A. Risse ${ }^{\dagger \dagger}$, \\ M. Risse ${ }^{\dagger}$, M. Roth ${ }^{*}$, G. Schatz ${ }^{\dagger}$, H. Schieler ${ }^{\dagger}$, H. Ulrich ${ }^{\dagger}$, J. van Buren ${ }^{\dagger}$, \\ A. Vardanyan ${ }^{\ddagger}$, A. Weindl ${ }^{\dagger}$, J. Wochele ${ }^{\dagger}$ and J. Zabierowski ${ }^{\dagger \dagger}$ \\ *Institut für Experimentelle Kernphysik, Universität Karlsruhe, 76021 Karlsruhe, Germany \\ ${ }^{\dagger}$ Institut für Kernphysik, Forschungszentrum Karlsruhe, 76021 Karlsruhe, Germany \\ **National Institute of Physics and Nuclear Engineering, 7690 Bucharest, Romania \\ ${ }^{\ddagger}$ Cosmic Ray Division, Yerevan Physics Institute, Yerevan 36, Armenia \\ ${ }^{\S}$ now at University of Wuppertal, 42097 Wuppertal, Germany \\ "now at University of Leeds, LS2 9JT Leeds, United Kingdom

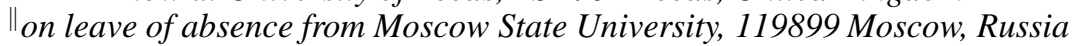 \\ ${ }^{\dagger}$ Soltan Institute for Nuclear Studies, 90950 Lodz, Poland
}

\begin{abstract}
Results of the KASCADE air shower experiment investigating the origin of cosmic rays in the energy region from $10^{13}$ to $10^{17} \mathrm{eV}$ are presented. Attention is drawn on the investigation of interactions in the atmosphere and the energy spectrum and mass composition of cosmic rays.
\end{abstract}

Keywords: cosmic rays; air shower; knee; high-energy interactions

PACS: 96.40.De; 96.40.Pq; 95.85.Ry; 13.85.-t

\section{INTRODUCTION}

One of the most remarkable structures in the energy spectrum of cosmic rays is a change of the spectral index $\gamma$ of the power law $d N / d E \propto E^{\gamma}$ at an energy of about $4 \mathrm{PeV}$, the so called knee [1, 2, 3]. The origin of the knee has not been resolved yet, however an explanation of the knee is thought to be a corner stone in the understanding of the origin of galactic cosmic rays. In the literature various reasons for the origin of the knee are discussed related to the acceleration and propagation processes of cosmic rays as well as to interactions in interstellar space or the Earth's atmosphere [4, 5].

The steeply falling energy spectrum requires large detection areas at high energies. The largest balloon borne experiment with single element resolution (TRACER, $5 \mathrm{~m}^{2} \mathrm{sr}$ ) reaches energies of a few $10^{14} \mathrm{eV}[\underline{6}]$. To study higher energies experiments covering several $10^{4} \mathrm{~m}^{2}$ and exposure times exceeding several years are necessary, which, at

\footnotetext{
${ }^{1}$ Invited talk, Astrophysical Sources of High Energy Particles and Radiation, Torun, June 20 - 24, 2005
} 
present, can only be realized in ground based installations. They measure the secondary products generated by high-energy cosmic-ray particles in the atmosphere - the extensive air showers. The challenge of these investigations is to reveal the properties of the shower inducing particle behind an absorber - the atmosphere - with a total thickness at sea level corresponding to 11 hadronic interaction lengths or 30 radiation lengths.

One of the most advanced experiments in the energy range from $10^{13} \mathrm{eV}$ to $10^{17} \mathrm{eV}$ is the experiment KASCADE ("KArlsruhe Shower Core and Array DEtector") [7]. It is continuously operating since 1996, detecting simultaneously the three main components of air showers. A $200 \times 200 \mathrm{~m}^{2}$ scintillator array measures the electromagnetic and muonic components $\left(E_{\mu}>0.23 \mathrm{GeV}\right)$. The central detector system combines a large hadron calorimeter, measuring the energy, as well as point and angle of incidence for hadrons with energies $E_{h}>50 \mathrm{GeV}$ [8], with several muon detection systems $\left(E_{\mu}>\right.$ $0.49,2.4 \mathrm{GeV})$ [9]. In addition, high-energy muons are measured by an underground muon tracking detector equipped with limited streamer tubes $\left(E_{\mu}>0.8 \mathrm{GeV}\right)$ [10]. The multi-detector set-up allows to address questions connected to the development of air showers in the atmosphere and the origin of high energy cosmic rays.

\section{HIGH-ENERGY INTERACTIONS}

Addressing astrophysical questions with air-shower data necessitates the understanding of high-energy interactions in the atmosphere. Or, in reversion, the interpretation of properties of primary radiation derived from air-shower measurements depend on the understanding of the complex processes during the development of air showers. Recent investigations indicate inconsistencies in the interpretation of air shower data [11, 12, 3]. Thus, one of the goals of KASCADE is to investigate high-energy interactions and to improve contemporary models to describe such processes.

For air shower interpretation the understanding of multi-particle production in hadronic interactions with a small momentum transfer is essential [13]. Due to the energy dependence of the coupling constant $\alpha_{s}$ soft interactions cannot be calculated within QCD using perturbation theory. Instead, phenomenological approaches have been introduced in different models. These models are the main source of uncertainties in simulation codes to calculate the development of extensive air showers, such as the program CORSIKA [14]. Several codes to describe hadronic interactions at low energies (GHEISHA, FLUKA, and UrQMD) as well as high energies (DPMJET, NEXUS, QGSJET, SIBYLL, and VENUS) have been embedded in CORSIKA. Their predictions are compared with experimental results in order to check their correctness.

Studies of the shower development in the atmosphere have been performed with the multi-detector set-up, see e.g. [15]. For example, deficiencies of some models have been pointed out [16], the lateral distributions of the electromagnetic, muonic, and hadronic components have been measured [17], and the attenuation and absorption lengths of showers have been determined [18].

Of particular importance for the interaction studies is the large hadron calorimeter, which has been calibrated recently at an accelerator beam with hadrons up to $350 \mathrm{GeV}$ [19]. It enables studies of the hadronic backbone of a shower. Interactions with small momentum transfer have been studied [20]. Such interactions are hardly accessible 

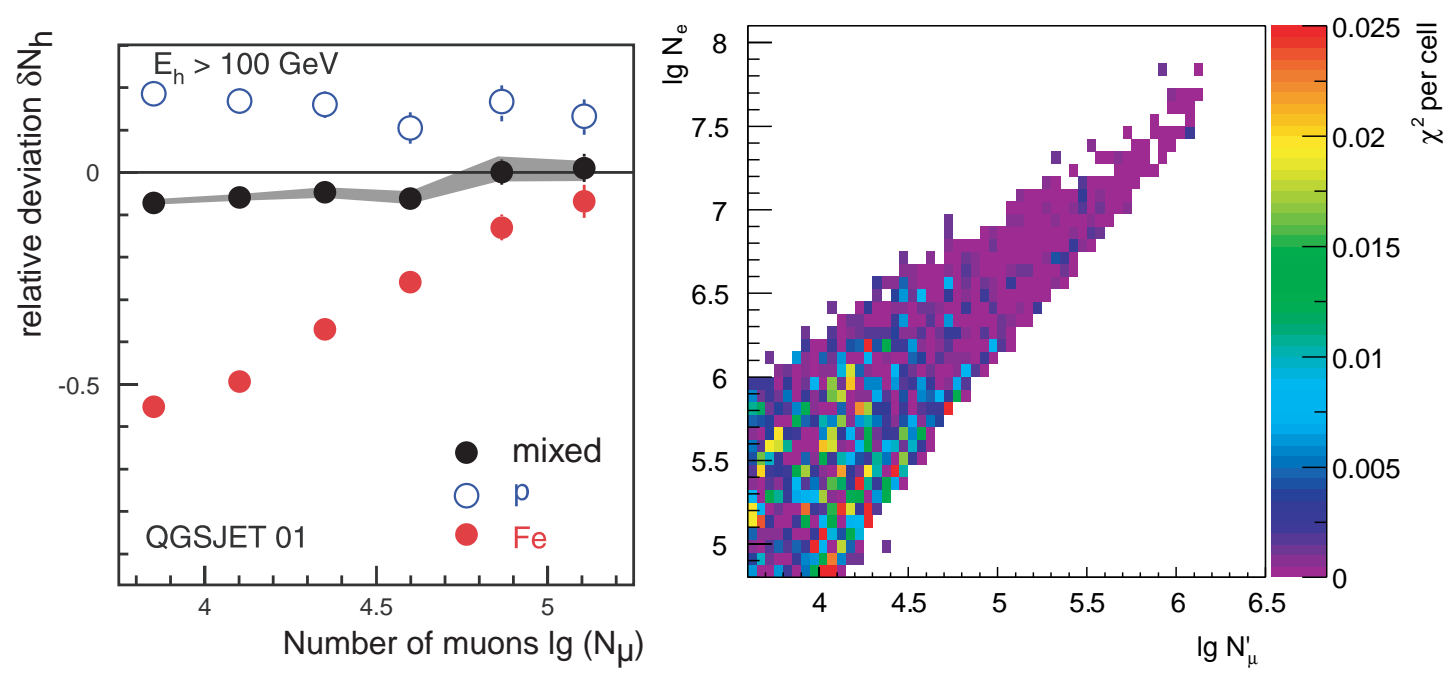

FIGURE 1. Left: Relative deviation of the number of reconstructed hadrons relative to the KASCADE measurements as function of the number of muons for simulations using CORSIKA/QGSJET 01, assuming pure protons and iron nuclei as well as a mixed composition [25]. Right: Deviation of the reconstructed and observed two-dimensional shower size spectrum using CORSIKA/QGSJET 01 to interpret the measurements, see text and [26].

at collider experiments (only at low energies in fixed target set-ups) and air-shower experiments provide complementary information. The arrival time of hadrons in air showers has been studied [21] and attention has been drawn on the investigation of the geometrical structure of the hadronic shower core to search for interactions with unusual large transverse momenta [22]. The transverse momentum of secondary pions in hadronic interactions is investigated by the reconstruction of the pseudo rapidity distributions of high-energy muons registered with the muon tracking detector [23].

A valuable tool to test high-energy interaction models are correlations between different shower components. Since the mass composition of cosmic rays is not well known in the $\mathrm{PeV}$ region, in such investigations frequently the measurements are compared to predictions for proton and iron induced showers as extreme assumptions for the composition. Such correlations are, for example, the dependence of the number of hadrons, the hadronic energy sum, or the energy of the most energetic hadron on the number of muons or electrons [24, 25]. A couple of years ago some models, like SIBYLL 1.6, DPMJET 2.5, or NEXUS 2 failed to describe the measurements in particular correlations. While on the other hand, for contemporary models, like QGSJET 01, SIBYLL 2.1, or DPMJET 2.55 the KASCADE measurements of various correlations of the electromagnetic, muonic, and hadronic components are bracketed by the extreme assumptions of primary protons and iron nuclei [24, 25].

While for previous analyses pure proton or iron compositions have been assumed, at present, more detailed analyses are performed [25]. They take into account the spectra for elemental groups as obtained from investigations of the electromagnetic and muonic components (see below). The relative deviation of the reconstructed number of hadrons $\delta N_{h}=\left(N_{h}^{\text {sim }}-N_{h}^{\text {meas }}\right) / N_{h}^{\text {meas }}$ for simulated values $N_{h}^{\text {sim }}$ relative to the KASCADE measurements $N_{h}^{\text {meas }}$ is shown in Fig. 1 (left) as function of the number of muons. Presented 
are values using CORSIKA/QGSJET 01 assuming protons, iron nuclei, and a mixed composition according to the five elemental groups. For a fully consistent model the values for the mixed composition are expected to agree with the measurements, i.e. should be at the zero line. One recognizes deviations of about $10 \%$ for low muon numbers $\left(\lg N_{\mu}<4.8\right)$, while the model yields consistent results for higher energies. It is remarkable that the same behavior has been observed for an analysis of the electromagnetic and muonic component [26], see below.

In conclusion, the models QGSJET 01 [27], SIBYLL 2.1 [28], and DPMJET 2.55 [29] seem to be the most reliable models to describe high-energy hadronic interactions. However, also they are not able to describe the data completely consistent [26]. This illustrates the resolution of experiments like KASCADE, which are able to study details of high-energy interactions in the atmosphere and indicates the progress made in this field during the last decade. At the same time, this stimulates new efforts, with the objective to improve the present interaction models. Examples are the study of airshower relevant parameters at accelerators [30], new theoretical concepts included in the QGSJET model [31], or the investigations of dedicated changes of physical parameters like the proton-proton cross-section or the inelasticity of hadronic interactions on airshower observables [32, 33]. The latter indicate that variations of interaction parameters within the error bounds of accelerator measurements yield significant and measurable changes in the air shower development [34].

\section{COSMIC-RAY ANISOTROPY}

Supernova remnants, such as Cassiopeia A, have been observed in electromagnetic radiation in a wide energy range up to $\mathrm{TeV}$-energies. Calculations indicate that the observed multi-wavelength spectra are consistent with the acceleration of cosmic-ray electrons and hadrons in supernova remnants [35]. Recent observations by the H.E.S.S. experiment reveal a shell structure of the supernova remnant RXJ-1713 and an energy spectrum of $\gamma$-rays $\propto E^{-2.2}$ in agreement with the idea of particle acceleration in a shock front [36]. The spectrum extends up to energies of $10 \mathrm{TeV}$ and provides evidence for the existence of particles with energies beyond $100 \mathrm{TeV}$ at the shock front that emerged from the supernova explosion. The $\gamma$-ray spectrum is expected to extend to much higher energies due to the production of neutral pions by charged cosmic rays interacting with the interstellar matter.

Also, of great interest is to study the arrival direction of charged cosmic rays to search for potential point sources. The arrival directions of showers with energies above $0.3 \mathrm{PeV}$ covering a region from $10^{\circ}$ to $80^{\circ}$ declination have been investigated with KASCADE [37]. No significant excess has been observed neither for all showers, nor for muon poor events. The analysis has been deepened by investigating a narrow band $\left( \pm 1.5^{\circ}\right)$ around the Galactic plane. Also circular regions around 52 supernova remnants and $10 \mathrm{TeV}-\boldsymbol{\gamma}$-ray sources have been studied. None of the searches provided a hint for a point source, neither by taking into account all events, nor selecting muon-poor showers only. Upper limits for the fluxes from point like sources are determined to be around $10^{-10} \mathrm{~m}^{-2} \mathrm{~s}^{-1}$. In addition, no clustering of the arrival direction for showers with primary energies above $80 \mathrm{PeV}$ is visible. 
While the search for point sources is related to the investigation of cosmic-ray acceleration sites, the large scale anisotropy is expected to reveal properties of the cosmic-ray propagation. The Rayleigh formalism is applied to the right ascension distribution of extensive air showers measured by KASCADE [38]. No hints of anisotropy are visible in the energy range from 0.7 to $6 \mathrm{PeV}$. This accounts for all showers, as well as for subsets containing showers induced by predominantly light or heavy primary particles. Upper limits for Rayleigh amplitudes are determined to be between $10^{-3}$ at a primary energy of $0.7 \mathrm{PeV}$ and $10^{-2}$ at $6 \mathrm{PeV}$. The increase of the amplitudes as function of energy is predicted by calculations using a diffusion model to describe the cosmic-ray propagation in the Galaxy [39]. This indicates that leakage from the Galaxy plays an important part during cosmic-ray propagation and most likely, the leakage is also (partly) responsible for the origin of the knee. On the other hand, simple Leaky-Box models describing cosmic-ray propagation seem to be ruled out by the measurements [38, 40, 41].

In addition, the data registered by the KASCADE experiment were searched for events which might be attributed to primary $\gamma$-rays [42]. Possible $\gamma$-induced events are identified by their low muon to electron ratios and by the steepness and smoothness of their electron lateral distributions. No local enhancement indicative of a point source was observed. In comparison with previous measurements, the upper limit could be improved and the range could be extended to higher energies.

\section{ENERGY SPECTRA AND MASS COMPOSITION}

The main objective of KASCADE is to determine the energy spectra and mass composition of cosmic rays. The problem has been approached from various points of view. It could be shown that a knee exists in all three main shower components, i.e. electrons, muons, and hadrons with a knee position at about 4 to $5 \mathrm{PeV}$ [43]. The primary energy spectrum could be established based on the electromagnetic and muonic [44] as well as the hadronic and muonic shower components [45]. Analyses of the electromagnetic and muonic shower components [46], the hadronic and muonic components [47], as well as various combinations of them [11] indicate an increase of the mean logarithmic mass of cosmic rays as function of energy in the knee region.

The longitudinal development of the muonic shower component is studied with the muon tracking detector of the KASCADE-Grande experiment [48]. The arrival direction and point of incidence of individual muons are registered and allow in conjunction with the geometric parameters of the electromagnetic shower the reconstruction of the average production height of muons. Through comparison of measured values with predicted production heights from Monte Carlo calculations, the mean logarithmic mass of cosmic rays is inferred. In the energy range from $\sim 0.4$ to $\sim 30 \mathrm{PeV}$ a steady increase of the mean logarithmic mass has been obtained.

Attention has also been drawn on a special class of events, the unaccompanied hadrons. These originate from small air showers, which have interacted at large heights and almost the complete shower has been absorbed in the atmosphere, and only one single hadron has been reconstructed in the calorimeter [49]. Simulations reveal that these events are closely related to the flux of primary protons. Hence, the measured flux of unaccompanied hadrons at ground level has been used to derive the spectrum of primary 

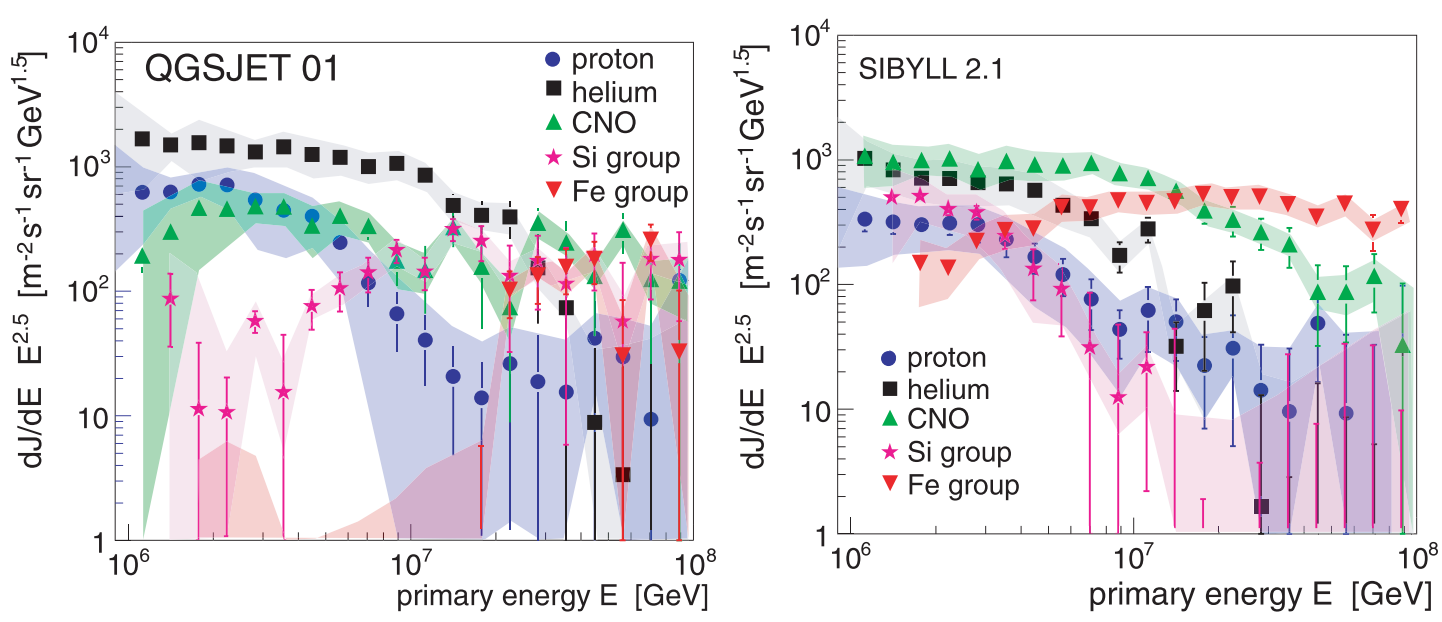

FIGURE 2. Energy spectra for five elemental groups using the hadronic interaction models QGSJET 01 (left) and SIBYLL 2.1 (right) to interpret the data [26].

protons based on simulations with CORSIKA/QGSJET. The resulting flux follows a single power law in the energy range from $100 \mathrm{GeV}$ to $1 \mathrm{PeV}$ and is compatible with direct measurements.

An advanced analysis is founded on the measurement of the electromagnetic and muonic shower components with the scintillator array [26]. The analysis is based on the deconvolution of the two-dimensional electron muon number distribution. The content of each cell in the $N_{e}-N_{\mu}$ plane is assumed to be the sum of contributions from five different primary elements, i.e. protons, helium, carbon, silicon, and iron. These elements are taken to represent the full spectrum of cosmic-ray nuclei.

The application of an unfolding procedure to the data is performed on basis of two hadronic interaction models (QGSJET 01 and SIBYLL 2.1) as implemented in the CORSIKA program. The resulting spectra for elemental groups are displayed in Fig. 2 The results exhibit sequential cut-offs for the individual mass groups for the proton, helium, and $\mathrm{CNO}$ components. The systematic differences for the spectra derived with QGSJET and SIBYLL amount to a factor of about two to three. The silicon and iron groups show a rather unexpected behavior for both models. The discrepancies are attributed to the fact that none of the models is able to describe the observed data set in the whole energy range consistently, for details see [26]. Assuming the obtained spectra in the simulations, the two dimensional shower size spectrum has been calculated and compared to the measurements. The differences in each cell of the $N_{e}-\mathrm{N}_{\mu}$ plane are depicted in Fig. 1 (right). One recognizes relatively large deviations at low energies $\left(\lg N_{\mu}<4.8\right)$, indicating inconsistencies for the model QGSJET 01 in this range. On the other hand, for SIBYLL 2.1 large differences are obtained at high energies.

Despite of the discrepancies, the data compare well to the results obtained by the EAS-Top experiment [50] and agree with extrapolations of direct measurements to high energies [51]. Considering the energy range above $10 \mathrm{GeV}$, at least a qualitative picture of the energy spectra for individual mass groups emerges. The spectra seem to be compatible with power laws with a cut-off at high energies. The cut-off behavior 
indicated by the measurements is reflected by theoretical considerations taking into account the maximum energy attained during acceleration in supernova remnants [52] or diffusive propagation of cosmic rays in the galactic magnetic fields [53].

\section{CONCLUSIONS AND OUTLOOK}

The KASCADE experiment, measuring simultaneously the electromagnetic, muonic, and hadronic shower components has reached the sensitivity to investigate details in the development of showers in the atmosphere and to give hints for the improvement of the description of hadronic interaction processes. With the KASCADE data hadronic interaction models could be improved within the last years. The all-particle energy spectrum is reasonably well known. For the first time, spectra for groups of elements could be derived in the knee region. The observed spectra seem to exhibit cut-offs proportional to the nuclear charge. The knee in the all-particle energy spectrum is caused by a depression of the flux of light elements. The increase of the mean logarithmic mass derived from air shower observations seems to be compatible with subsequential cut-offs for individual elements.

Energy spectra have been reconstructed with KASCADE data up to energies of $100 \mathrm{PeV}$. At these energies statistical errors start to dominate the overall error. To improve this situation the experiment has been enlarged. Covering an area of $0.5 \mathrm{~km}^{2}$, 37 detector stations, containing $10 \mathrm{~m}^{2}$ of plastic scintillators each, have been installed around the original KASCADE set-up [54]. Regular measurements with this new array and the original KASCADE detectors, forming the KASCADE-Grande experiment, are performed since summer 2003. First analyses extend the lateral distributions of electrons and muons up to $600 \mathrm{~m}[55,56]$. The objective is to reconstruct primary energy spectra for elemental groups up to $10^{18} \mathrm{eV}$ [57], covering the energy region of the second knee, where the galactic cosmic ray spectrum is expected to end [41].

A new technique to measure air showers is about to be established: The LOPES experiment, consisting of 30 dipole antennas, operated at the site of the KASCADE experiment, has registered first air showers, observing radio emission in the frequency range from 40 to $80 \mathrm{MHz}$ [58]. Analyses show a correlation between the strength of the radio signal and the angle between the shower axis and the direction of the geomagnetic field, indicating that the emission process is most likely geosynchrotron emission. An increase of the detected radio signal as function of the shower energy has been observed. The new technique is expected to contribute to the exploration of air showers at energies exceeding $10^{17} \mathrm{eV}$ through the measurement of the longitudinal shower development.

KASCADE is supported by the Ministry for Research and Education of Germany,

the Polish State Committee for Scientific Research (KBN grant for 2004-06) and the Romanian National Academy for Science, Research and Technology.

\section{REFERENCES}

1. M. Nagano and A. Watson, Rev. Mod. Phys., 72, 689 (2000).

2. A. Haungs et al., Reports on Progress in Physics, 66, 1145 (2003).

3. J.R. Hörandel, Astropart. Phys., 19, 193 (2003). 
4. J.R. Hörandel, Astropart. Phys., 21, 241 (2004).

5. J.R. Hörandel, astro-ph/0501251 (2005).

6. D. Müller et al., Proc. 29th Int. Cosmic Ray Conf., Pune (2005).

7. T. Antoni et al., Nucl. Instr. \& Meth. A, 513, 490 (2003).

8. J. Engler et al., Nucl. Instr. \& Meth. A, 427, 528 (1999).

9. H. Bozdog et al., Nucl. Instr. \& Meth. A, 465, 455 (2001).

10. P. Doll et al., Nucl. Instr. \& Meth. A, 488, 517 (2002).

11. T. Antoni et al., Astropart. Phys., 16, 245 (2002).

12. S. Swordy et al., Astropart. Phys., 18, 129 (2002).

13. R. Engel, astro-ph/0504358 (2005).

14. D. Heck et al., Report FZKA 6019, Forschungszentrum Karlsruhe (1998).

15. J.R. Hörandel, Nucl. Phys. B (Proc. Suppl.), 122, 455 (2003).

16. T. Antoni et al., J. Phys. G: Nucl. Part. Phys., 25, 2161 (1999).

17. T. Antoni et al., Astropart. Phys., 14, 245 (2001).

18. T. Antoni et al., Astropart. Phys., 19, 703 (2003).

19. S. Plewnia et al., Calorimetry in Particle Physics, C. Cecchi, P. Cenci, P. Lubrano, M. Pepe (eds.), World Scientific, 2005, p. 199.

20. T. Antoni et al., J. Phys. G: Nucl. Part. Phys., 27, 1785 (2001).

21. J.R. Hörandel et al., Nucl. Phys. B (Proc. Suppl.), 122, 309 (2003).

22. T. Antoni et al., Phys. Rev. D, 71, 072002 (2005).

23. J. Zabierowski et al., Nucl. Phys. B (Proc. Suppl.), (Proc. 13th ISVHECRI) in press (2005).

24. J. Milke et al., Acta Physica Polonica B, 35, 341 (2004).

25. J. Milke et al., Proc. 29th Int. Cosmic Ray Conf., Pune (2005).

26. T. Antoni et al., astro-ph/0505413 Astropart. Phys. in press (2005).

27. N. Kalmykov et al., Nucl. Phys. B (Proc. Suppl.), 52B, 17 (1997).

28. R. Engel et al., Proc. 26th Int. Cosmic Ray Conf., Salt Lake City, 1, 415 (1999).

29. J. Ranft, Phys. Rev. D, 51, 64 (1995).

30. C. Meurer et al., astro-ph/0506755 (2005).

31. S. Ostapchenko, astro-ph/0412591 (2005).

32. J.R. Hörandel, J. Phys. G: Nucl. Part. Phys., 29, 2439 (2003).

33. J.R. Hörandel et al., Nucl. Phys. B (Proc. Suppl.), (Proc. 13th ISVHECRI) in press (2005).

34. J.R. Hörandel et al., Proc. 29th Int. Cosmic Ray Conf., Pune (2005).

35. E. Berezhko et al., Astron. \& Astroph., 400, 971 (2003).

36. F. Aharonian et al., Nature, 432, 75 (2004).

37. T. Antoni et al., Astrophys. J., 608, 865 (2004).

38. T. Antoni et al., Astrophys. J., 604, 687 (2004).

39. J. Candia et al., J. Cosmol. Astropart. Phys., 5, 3 (2003).

40. G. Maier et al., Int. J. Mod. Phys. A, in press (2005).

41. J.R. Hörandel et al., astro-ph/0508015 (2005).

42. G. Schatz et al., Proc. 28th Int. Cosmic Ray Conf., Tsukuba, 4, 2293 (2003).

43. R. Glasstetter et al., Nucl. Phys. B (Proc. Suppl.), 75A, 238 (1999).

44. R. Glasstetter et al., Proc. 26th Int. Cosmic Ray Conf., Salt Lake City, 1, 222 (1999).

45. J.R. Hörandel et al., Proc. 26th Int. Cosmic Ray Conf., Salt Lake City, 1, 337 (1999).

46. J. Weber et al., Proc. 26th Int. Cosmic Ray Conf., Salt Lake City, 1, 341 (1999).

47. J.R. Hörandel et al., Proc. 16th European Cosmic Ray Symposium, Alcala de Henares, p. 579 (1998).

48. C. Büttner et al., Proc. 28th Int. Cosmic Ray Conf., Tsukuba, 1, 33 (2003).

49. T. Antoni et al., Astrophys. J., 612, 914 (2004).

50. G. Navarra et al., Proc. 28th Int. Cosmic Ray Conf., Tsukuba, 1, 147 (2003).

51. J.R. Hörandel, astro-ph/0508014(2005).

52. L. Sveshnikova et al., Astron. \& Astroph., 409, 799 (2003).

53. N. Kalmykov and A. Pavlov, Proc. 26th Int. Cosmic Ray Conf., Salt Lake City, 4, 263 (1999).

54. G. Navarra et al., Nucl. Instr. \& Meth. A, 518, 207 (2004).

55. R. Glasstetter et al., Proc. 29th Int. Cosmic Ray Conf., Pune (2005).

56. J. v. Buren et al., Proc. 29th Int. Cosmic Ray Conf., Pune (2005).

57. A. Haungs et al., astro-ph/0508286 (2005).

58. H. Falcke et al., Nature, 435, 313 (2005). 